\title{
CD8-Positive T cells and CD204-Positive M2 Macrophages Predict Postoperative Prognosis of Very High-Risk Prostate Cancer
}

\section{Yoshinori Yanai}

Keio University School of Medicine

Takeo Kosaka ( $\nabla$ takemduro@gmail.com )

Keio University School of Medicine

Shuji Mikami

Keio University School of Medicine

Hiroshi Hongo

Keio University School of Medicine

Yota Yasumizu

Keio University School of Medicine

Toshikazu Takeda

Keio University School of Medicine

Kazuhiro Matsumoto

Keio University School of Medicine

Jun Miyauchi

Saitama City Hospital

Shigehisa Kitano

National Cancer Center Hospital

Mototsugu Oya

Keio University School of Medicine

\section{Research Article}

Keywords: Biochemical recurrence, CD8+ tumor-infiltrating immune cells, CD204+ M2 macrophages, Prostate cancer, Radical prostatectomy, Seminal vesicle invasion

Posted Date: August 26th, 2021

DOl: https://doi.org/10.21203/rs.3.rs-820466/v1

License: (c) (1) This work is licensed under a Creative Commons Attribution 4.0 International License. Read Full License 


\section{Abstract}

To stratify the heterogeneity of prostate cancer (PCa) with seminal vesicle invasion (SVI) immunologically after radical prostatectomy (RP) focusing on the tumor microenvironment. We retrospectively reviewed the clinicopathological data of $71 \mathrm{PCa}$ patients with SVI, which is known as a factor of very high risk PCa. Preoperative clinical variables and postoperative pathological variables were evaluated as predictors of BCR with a multivariate logistic regression. Immune cell infiltration including the CD8-positive cell (CD8 ${ }^{+}$cell) and CD204-positive M2 macrophage (CD204 ${ }^{+}$cell) was investigated by immunohistochemistry. The cumulative incidence and risk of BCR were assessed with a Kaplan-Meier analysis and competing risks regression. A higher $\mathrm{CD} 8^{+}$cell count in the SVI area significantly indicated a favorable prognosis for cancers with SVI $(p=0.004)$. A lower CD $204^{+}$cell count in the SVI area also significantly indicated a favorable prognosis for cancers with SVI $(p=0.004)$. Furthermore, the combination of the $\mathrm{CD}^{+}$and $\mathrm{CD} 204^{+}$cell infiltration ratio of the SVI area to the main tumor area was a significant factor for BCR in the patients with the PCa with SVI $(p=0.001)$. In PCa patients with SVI, the combination of $\mathrm{CD}^{+}$and $\mathrm{CD} 204^{+}$cell infiltration is useful to predict the prognosis.

\section{Introduction}

Some very high-risk prostate cancer (PCa) patients after radical prostatectomy (RP) experience long-term survival without biochemical recurrence (BCR). BCR is generally defined as the asymptomatic increase of serum prostate-specific antigen (PSA) levels after RP to greater than $0.2 \mathrm{ng} / \mathrm{ml}$ [1]. A high Gleason score, positive surgical margins, maximum index tumor diameter, extraprostatic extension (EPE), and seminal vesicle invasion (SVI) of PCa were significantly associated with BCR [2][3][4]. However, some patients with SVI experience long-term survival without BCR after RP. No clinical and pathological characteristics have been accurately stratified in such patients yet.

Although prostate tumor cells are surrounded by a complex tumor microenvironment including host inflammatory or immune effectors, the contribution of the tumor immune response remains unknown [5]. We previously reported that CD204 positive M2 macrophages (CD204 ${ }^{+}$cell) are associated with prognosis in thymic carcinoma. In that report, CD8 and CD204 positive cells in stroma were identified as possible better prognostic biomarkers, considering the heterogeneity of other biomarkers [6]. To better understand the immune profile within prostate tumor local progression, we focused on the two different type of immune cells and the marker localization. One is CD8-positive effector T cell (CD8 ${ }^{+}$cell) as effector cells that play the most important role in the anti-tumor immune response. The other is CD204positive M2 macrophage, which is reported as one of the representative negative prognostic factors [7]. Macrophages can be classified into M1 and M2 subtypes, and CD204 is a marker of M2 macrophages and plays important roles in the tumor microenvironment by inhibiting anti-tumor immune responses [6] [8]. However, there were no study concerning the two different type of immune cells and the marker localization in prostate cancer with SVI. We hypothesized that infiltration patterns of $\mathrm{CD}^{+}$cell and $\mathrm{CD} 204^{+}$cell in the SVI area might differ from those in the main tumor area. The aim of this study was to 
investigate the clinical, pathological and immunological significance of the tumor immune microenvironment of very high-risk PCa with SVI.

\section{Materials And Methods Patients}

We retrospectively reviewed the medical records of 1,286 patients who underwent RP at Keio University Hospital and Saitama City hospital from January 2005 to December 2018. The patients' serum PSA levels were assessed after RP. A postoperative PSA level greater than $0.2 \mathrm{ng} / \mathrm{ml}$ was defined as BCR. Inclusion criteria was SVI and we excluded patients who were lacking data. 7 patients who received adjuvant therapy and 8 patients without a nadir PSA level less than $0.2 \mathrm{ng} / \mathrm{ml}$ were also excluded. Finally, we analyzed 71 (5.5\%) patients. This study was approved by the Institutional Review Board of Keio University and Saitama City Hospital. Informed written consent was obtained from the study participants.

\section{Pathological analysis}

The Gleason scores after the RP were cited from pathological reports. In patients who received neoadjuvant androgen deprivation therapy, Gleason scores obtained from a needle biopsy were used instead of prostatectomy Gleason scores. All PCa cases were histologically diagnosed before prostatectomy by ultrasound-guided needle biopsy via the transrectal or transperineal approach. After RP, all specimens were fixed in $10 \%$ formalin and embedded in paraffin. Whole-mount section pathology was performed, and all specimens were cut into thin slices perpendicular to the urethra from the apex to the base after removing the seminal vesicle. All seminal vesicles were cut longitudinally. All slides were stained with hematoxylin and eosin. The Gleason score of the PCa and the other pathological parameters, including SVI, were evaluated in each section. SVI was defined as cancer invasion into the extraprostatic portion of the seminal vesicles.

\section{Immunohistochemistry}

Immunohistochemistry was performed after the sections were formalin-fixed and paraffin-embedded. The sections were deparaffinized in xylene and then rehydrated in graded alcohols and distilled water. After antigen retrieval with citric acid ( $\mathrm{pH} 6.0$ ), endogenous peroxidase activity was blocked with $1 \%$ hydrogen peroxide for 30 minutes, followed by washing with distilled water. To bind nonspecific antigens, the sections were incubated with $5 \%$ skim milk for 15 minutes. The sections were incubated with an anti-CD 8 rabbit polyclonal antibodies (1:100 dilation, Abcam, Cambridge, MA, USA) and anti-CD204 rabbit polyclonal antibodies (1:100 dilation, Abcam, Cambridge, MA, USA) at room temperature for one hour, followed by conjugation to the secondary antibody and DAB staining.

\section{Evaluation of immunostaining}

To evaluate CD8 and CD204 staining, the cells with positive staining were counted in five representative fields $\left(200 \mu \mathrm{m}^{2} /\right.$ field) using light microscopy. The median number of the CD $8^{+}$and $\mathrm{CD} 204^{+}$cell was 
estimated for each main tumor area and SVI area. Values above median number of the CD $8^{+}$or $\mathrm{CD} 204^{+}$ cells were considered as CD8-high or CD204-high. Values under median number of the $\mathrm{CD}^{+}$or $\mathrm{CD} 204^{+}$ cells were considered as CD8-low or CD204-low. The experienced urologic pathologists, blinded to the patients' clinical data, performed the counting.

\section{SVM (the Seminal Vesicle area to the Main area) Score}

Focusing on the distribution of the $\mathrm{CD}^{+}$and $\mathrm{CD} 204^{+}$cells in each tumor slide, we calculated the ratio of the $\mathrm{CD}^{+}$and $\mathrm{CD} 204^{+}$cell counts in the SVI area to those in the main tumor area for each patient. We defined the ratio as "SVM (the Seminal Vesicle area to the Main area) Score". We calculated estimates (with confidence intervals) of the best-fit receiver operating characteristic (ROC) curve and the corresponding area under the ROC curve (AUC), and then calculated the cutoff value of each SVM Score for $\mathrm{CD} 8^{+}$and $\mathrm{CD} 204^{+}$cells, respectively.

\section{Statistical analysis}

Differences in continuous variables between groups were evaluated using the Mann-Whitney $U$ test. The Chi-squared test was used to analyze the difference in the number of patients between two groups. To identify factors predictive of BCR, univariate and multivariate analyses were performed using the Cox proportional hazards model with stepwise forward selection. Kaplan-Meier curves were drawn to evaluate postoperative BCR-free survival. All reported p-values were two-sided, and statistical significance was set at 0.05 . The statistical analyses were performed using the R Statistical Language version 3.5.3 program and the SPSS version 25.0 statistical software package.

\section{Results}

\section{Patients characteristics of seminal vesicle invasion}

The clinicopathological data of the 71 patients with SVI are summarized in Table 1. The median PSA value at biopsy was $9.1 \pm 8.6 \mathrm{ng} / \mathrm{ml}$. The median prostate volume at biopsy was $30.0 \pm 12.2 \mathrm{ml}$. The median PSA density at biopsy was $0.36 \pm 0.51 \mathrm{ng} / \mathrm{ml} / \mathrm{ml}$. SVI was not detected (clinical T3b) in any patients before RP. 
Table 1

Patient's characteristics

\begin{tabular}{|c|c|c|}
\hline & $\mathbf{N}$ & Median \pm SD $(5-95 \% \mathrm{Cl})$ \\
\hline Age at operation (yr) & & $68.4 \pm 5.7(55.5-75.3)$ \\
\hline$<66$ & $24(33.8 \%)$ & \\
\hline$\geqq 66$ & $47(66.2 \%)$ & \\
\hline PSA value at biopsy (ng/ml) & & $9.1 \pm 8.6(4.7-32.5)$ \\
\hline$<10$ & $39(54.9 \%)$ & \\
\hline $10-20$ & $23(32.4 \%)$ & \\
\hline$>20$ & $8(11.3 \%)$ & \\
\hline unknown & $1(1.4 \%)$ & \\
\hline Prostate volume at biopsy (ml) & & $30.0 \pm 12.2(13.7-51.9)$ \\
\hline$<30$ & $34(47.9 \%)$ & \\
\hline$\geqq 30$ & $34(47.9 \%)$ & \\
\hline unknown & $3(4.2 \%)$ & \\
\hline PSA-density at biopsy $(\mathrm{ng} / \mathrm{ml} / \mathrm{ml})$ & & $0.36 \pm 0.51(0.12-1.18)$ \\
\hline$<0.20$ & $15(21.1 \%)$ & \\
\hline$\geqq 0.20$ & $53(74.6 \%)$ & \\
\hline unknown & $3(4.2 \%)$ & \\
\hline \multicolumn{3}{|l|}{ clinical T stage } \\
\hline$c T 1 c, 2 a, 2 b$ & $47(66.2 \%)$ & \\
\hline cT2c, 3a & $22(31.0 \%)$ & \\
\hline unknown & $2(2.8 \%)$ & \\
\hline \multicolumn{3}{|l|}{ Grade Group } \\
\hline 1,2 & $14(19.7 \%)$ & \\
\hline $3,4,5$ & 57 (80.3\%) & \\
\hline
\end{tabular}

\section{Immune cell infiltration in prostate tissue}

The median $\mathrm{CD}^{+}$cell count in the main tumor area was $40.0 \pm 75.4(95 \% \mathrm{Cl}: 5.0-189.0)$ cells $/ \mathrm{mm}^{2}$ compared with $30.0 \pm 42.7$ (95\% Cl:3.75-150.0) cells $/ \mathrm{mm}^{2}$ in the SVI area. No significant difference was observed in BCR between the higher and lower $C D 8^{+}$cell count in the main tumor area $(p=0.401$, Fig. 1a). 
However, the statistically significant difference was observed in BCR between the higher and lower CD $8^{+}$ cell count in the SVI area, which suggested that a higher $\mathrm{CD} 8^{+}$cell count in the main tumor area predicted a favorable prognosis for PCa with SVI ( $p=0.004$, Fig. 1b).

The median CD204 ${ }^{+}$cell count in the main tumor area and the SVI area was $300.0 \pm 139.9$ (95\% Cl: $50.0-$ $540.0)$ cells $/ \mathrm{mm}^{2}$ and $300.0 \pm 163.1$ (95\% Cl: $\left.25.0-600.0\right)$ cells $/ \mathrm{mm}^{2}$, respectively. The statistically significant difference was observed in BCR between the higher and lower CD204 ${ }^{+}$cell count in the main tumor area, which suggested that a higher $\mathrm{CD} 204^{+}$cell count in the main tumor area predicted a worse prognosis for PCa with SVI ( $p=0.042$, Fig. $1 c)$. In the SVI area, the statistically significant difference was also observed in BCR between the higher and lower CD204+ cell count ( $p=0.004$, Fig. $1 d)$.

\section{Combination of immune cell infiltration}

The combination of the $\mathrm{CD} 8^{+}$and $\mathrm{CD} 204^{+}$cells in the main tumor area and SVI area was evaluated in each patients. Each patients were classified into four groups according to the amount of the $\mathrm{CD} 8^{+}$and CD204 ${ }^{+}$cells. Group I included patients with a higher $\mathrm{CD} 8^{+}$cell count and a lower $\mathrm{CD} 204^{+}$cell count. Group II included patients with a higher $\mathrm{CD} 8^{+}$cell count and a higher $\mathrm{CD} 204^{+}$cell count. Group III included patients with a lower $\mathrm{CD} 8^{+}$cell count and a lower CD $204^{+}$cell count. Group IV included patients with a lower $\mathrm{CD} 8^{+}$cell count and a higher $\mathrm{CD} 204^{+}$cell count. No significant difference among four groups was observed in the main tumor area ( $p=0.150$, Fig. 2a). However, a significant difference between four groups was observed in the SVI area ( $p<0.001$, Fig. $2 b)$. Group I had the most favorable outcome among the four groups. The 5-year progression free survival (PFS) rate of Group I was $90.9 \%$. Group II and Group III had the intermediate outcome. The 5-year PFS rate of Group II and III was $66.7 \%$ and $50.0 \%$, respectively. Group IV had the worst outcome. The 5-year PFS rate of Group IV was $25.0 \%$.

\section{Logistic regression analysis}

Univariate Cox regression revealed the serum PSA level, the CD8 ${ }^{+}$cell count in the SVI area and the CD204 ${ }^{+}$cell count in the SVI area had significant impact on BCR $(p=0.001, p=0.002$ and $p=0.036$, respectively, Table 2). Among the clinicopathologic parameters analyzed, multivariate Cox regression revealed that the higher serum PSA level (Hazard Ratio $(H R)=2.870, p=0.004)$, the lower $C D 8^{+}$cell count $(H R=3.004, p=0.018)$ and the higher $C D 204^{+}$cell count in the SVI area $(H R=3.290, p=0.013)$ are an indication of the unfavorable prognosis of cancers with SVI (Table 2). 
Table 2

Biochemical recurrence free survival according to Cox proportional hazards analysis

\begin{tabular}{|c|c|c|c|c|c|}
\hline \multirow[t]{2}{*}{ Variable } & \multirow[t]{2}{*}{ Univariate } & \multicolumn{4}{|c|}{ Multivariate } \\
\hline & & $\begin{array}{l}\text { Hazard } \\
\text { ratio }\end{array}$ & $5 \% \mathrm{Cl}$ & $95 \% \mathrm{Cl}$ & $\begin{array}{l}\text { p- } \\
\text { value }\end{array}$ \\
\hline Age at operation (yr) & 0.115 & & & & \\
\hline PSA value at biopsy $(\mathrm{ng} / \mathrm{ml})$ & 0.001 & 2.870 & 1.399 & 5.889 & 0.004 \\
\hline Grade Group & 0.371 & & & & \\
\hline $\mathrm{CD}^{+}$cell count in the main tumor area & 0.469 & & & & \\
\hline $\begin{array}{l}\mathrm{CD} 8^{+} \text {cell count in the seminal vesicle invasion } \\
\text { area }\end{array}$ & 0.002 & 3.004 & 1.207 & 7.478 & 0.018 \\
\hline $\mathrm{CD} 24^{+}$cell count in the main tumor area & 0.198 & & & & \\
\hline $\begin{array}{l}\mathrm{CD}^{2} 24^{+} \text {cell count in the seminal vesicle } \\
\text { invasion area }\end{array}$ & 0.036 & 3.290 & 1.282 & 8.443 & 0.013 \\
\hline
\end{tabular}

\section{SVM (the Seminal Vesicle area to the Main area) Score}

Each cut-off value of SVM Score of $\mathrm{CD}^{+}$and $\mathrm{CD} 204^{+}$cell was 1.5 and 0.6 , respectively. A statistically significant difference in the SVM Score of the CD8 $8^{+}$cell was observed $(p=0.012$, Fig. 3a). It suggested that the higher SVM Score of the CD ${ }^{+}$cell predicted a favorable prognosis of PCa with SVI. A statistically significant difference in the SVM Score of the CD204 ${ }^{+}$cell was also observed ( $p=0.037$, Fig. $\left.3 b\right)$. It suggested that the lower SVM Score of the CD204+ cell predicted a favorable prognosis of PCa with SVI. A statistically significant difference in the combination of the SVM Score of the CD8 ${ }^{+}$and $C D 204^{+}$cell was observed among the four groups $(p=0.002$, Fig. $3 c)$.

\section{Discussion}

SVI is an independent prognostic factor of $\mathrm{PCa}$, and in general, adjuvant radiotherapy is effective in patients with SVI [9]. However, this study revealed that the higher $\mathrm{CD} 8^{+}$cell count and the lower CD204 ${ }^{+}$ cell count in the SVI area are an indication of the favorable prognosis of cancers with SVI.

Several studies have been reported that $\mathrm{CD} 8^{+}$cell infiltration is associated with better prognosis [10][11]. In other reports, $\mathrm{CD} 8^{+}$cell was also associated with recurrence, progression, or lymph node invasion [12] [13]. $C D 8^{+}$cell may be associated with cancer progression by inhibiting the activity of effector T cells [14] [15]. Thus, we had hypothesized that higher infiltration of $C D 8^{+}$cell could be a favorable prognostic factor of BCR, however, no significant difference was observed in BCR between the higher and lower CD8 ${ }^{+}$ 
cell count in the main tumor area (Fig. 1a). Then, we focused on the distribution of the $\mathrm{CD} 8^{+}$cell in each tumor slide. $\mathrm{CD}^{+}$cell secret cytotoxic molecules and cytokines, including perforin, granzyme, interferongamma (IFN- $\gamma$ ), tumor necrosis factor-alpha and so on, which elicit anti-tumor effects. They are released only in the direction of the target cell by identifying cancer antigen-derived epitopes presented on $\mathrm{MHC}$ molecules on tumor cells by $T$ cell receptors to avoid non-specific bystander damage to normal tissue [16]. The mechanism might suggest our data that $\mathrm{CD} 8^{+}$cell increased in the SVI area rather than the main tumor area in each unfavorable tumors was correct (Fig. 1b).

On the other hand, tumor-associated macrophage (TAM) infiltration in the tumor microenvironment is directly associated with tumor invasion, nodal status, and clinical stage in some cancers, and TAMs also have been reported to regulate the growth of prostate cancer [17][18][19]. A previous study reported that M2 TAMs inhibit cytotoxic CD8 ${ }^{+}$cell, resulting in weakened anti-tumor immunity and the CD204 silencingenhanced tumor cell death was associated with elevated IFN- $\gamma$ levels in tumor tissue and increased tumor-infiltrating $\mathrm{CD}^{+}$cells [20]. We also focused on the distribution of $\mathrm{CD} 204^{+}$cell in each tumor slide as well as $\mathrm{CD}^{+}$cell. In our present study, a significant difference between the higher and lower CD204 ${ }^{+}$ cell count in the main tumor area and the SVI area was observed in BCR (Fig. 1c and 1d).

Patients with very high risk PCa, including SVI are usually considered to be candidates for adjuvant therapy [21][22][23]. However, it is uncertain whether all patients need adjuvant therapy, as some of them do not appear to benefit. Therefore, the development of an improved prediction model of the very high risk PCa is necessary and unmet needs. In this study, we assessed the relationship of the distribution of the $\mathrm{CD} 8^{+}$and $\mathrm{CD} 204^{+}$cell. The combination of those different immune cells could be more useful for predicting the prognosis of the very high risk PCa than a single immune cell. This study was derived from a retrospective review of patients treated at two institutions and the specimens used in this study could not reflect all status of the tumor immune microenvironment. The small tumor volume in SVI is also difficult to evaluate. However, the findings obtained in this study could be useful for clinical assessment and decision-making for PCa patients with SVI after RP. It is highly worthwhile to verify these results focused on the different type of immune cells and the marker localization in future prospective studies.

In conclusion, in prostate cancer patients with SVI, the combination of the $\mathrm{CD} 8^{+}$and $\mathrm{CD} 204^{+}$cell infiltration in the SVI area is useful to predict the prognosis.

\section{Declarations}

Acknowledgments. We thank Yoko Suzuki, Emmy Yanagida, and Hiroshi Yamada for technical assistance.

\section{Funding}

This work was supported by Grants-in-Aid for Scientific Research (\#17K11158 to Kosaka, and \#18K16709 to Yanai) and Takeda Science Foundation (to Kosaka), Japan from the Ministry of Education, 
Culture, Sports, Science, and Technology of Japan, and from SGH foundation (to Kosaka), Japan.

\section{Conflict of Interest and Disclosure Statement}

All authors have no financial disclosures to report.

Ethics approval and consent to participate: Written informed consent was obtained from all patients included in the study. This study was approved by the Institutional Review Board of Keio University and Saitama City Hospital and was performed in accordance with the Declaration of Helsinki.

Authors' contributions: $\mathrm{YY}$ and TK contributed to the conception and design of the study. YY, TK, TT, KM and SK drafted the manuscript. YY, TK, YY, HH and MO contributed to the acquisition, analysis of data and contributed statistical review of data. All authors took part

in interpretation of data. SM and JM performed pathological evaluation. All authors contributed to critical revision of the manuscript for important intellectual content. All authors read and approved the final manuscript.

\section{References}

1. Cookson MS, Aus G, Burnett AL, et al. Variation in the definition of biochemical recurrence in patients treated for localized prostate cancer: the American Urological Association Prostate Guidelines for Localized Prostate Cancer Update Panel report and recommendations for a standard in the reporting of surgical outcomes. J Urol. 177, 540-545 (2007).

2. Mizuno R, Nakashima J, Mukai M, et al. Tumour length of the largest focus predicts prostate-specific antigen-based recurrence after radical prostatectomy in clinically localized prostate cancer. $B J U$ Int. 104, 1215-1218 (2009).

3. Takamatsu K, Matsumoto K, Shojo K, et al. The prognostic value of zonal origin and extraprostatic extension of prostate cancer for biochemical recurrence after radical prostatectomy. Urol Oncol Semin Orig Investig. 37, 575.e19-575.e25 (2019).

4. Swanson GP, Riggs M, Hermans M. Pathologic findings at radical prostatectomy: Risk factors for failure and death. Urol Oncol Semin Orig Investig. 25, 110-114 (2007).

5. Fay EK, Graff JN. Immunotherapy in prostate cancer. Cancers (Basel). 12, 1-17 (2020).

6. Sato J, Kitano S, Motoi N, et al. CD20+ tumor-infiltrating immune cells and CD204+ $\mathrm{M} 2$ macrophages are associated with prognosis in thymic carcinoma. Cancer Sci. 111, 1921-1932 (2020).

7. Bates GJ, Fox SB, Han C, et al. Quantification of regulatory T cells enables the identification of highrisk breast cancer patients and those at risk of late relapse. J Clin Oncol. 24, 5373-5380 (2006).

8. Kawachi A, Yoshida H, Kitano S, Ino Y, Kato T, Hiraoka N. Tumor-associated CD204 ${ }^{+} \mathrm{M} 2$ macrophages are unfavorable prognostic indicators in uterine cervical adenocarcinoma. Cancer Sci. 109, 863-870 (2018). 
9. Swanson GP, Goldman B, Tangen CM, et al. The prognostic impact of seminal vesicle involvement found at prostatectomy and the effects of adjuvant radiation: Data from Southwest Oncology Group 8794. J Urol. 180, 2453-2458 (2008).

10. Hartman DJ, Frank M, Seigh L, et al. Automated quantitation of CD8-positive T Cells predicts prognosis in colonic adenocarcinoma with mucinous, signet ring cell, or medullary differentiation independent of mismatch repair protein status. Am J Surg Pathol. 44, 991-1001 (2020).

11. Lu J, Xu Y, Wu Y, et al. Tumor-infiltrating $C D 8^{+} T$ cells combined with tumor-associated $C D 68^{+}$ macrophages predict postoperative prognosis and adjuvant chemotherapy benefit in resected gastric cancer. BMC Cancer. 19, 1-10 (2019).

12. Leclerc BG, Charlebois $R$, Chouinard G, et al. CD73 expression is an independent prognostic factor in prostate cancer. Clin Cancer Res. 22, 158-166 (2006).

13. Petitprez F, Fossati N, Vano Y, et al. PD-L1 Expression and CD8 ${ }^{+}$T-cell Infiltrate are associated with clinical progression in patients with node-positive prostate cancer. Eur Urol Focus. 5, 192-196 (2019).

14. Becht E, Giraldo NA, Germain C, et al. Immune Contexture, Immunoscore, and Malignant Cell Molecular Subgroups for Prognostic and Theranostic Classifications of Cancers. Adv Immunol. 130, 95-190 (2016).

15. Horton BL, Williams JB, Cabanov A, Spranger S, Gajewski TF. Intratumoral CD8 ${ }^{+}$T-cell apoptosis is a major component of T-cell dysfunction and impedes antitumor immunity. Cancer Immunol Res. 6, 14-24 (2018).

16. Spranger S, Koblish HK, Horton B, Scherle PA, Newton R, Gajewski TF. Mechanism of tumor rejection with doublets of CTLA-4, PD-1/PD-L1, or IDO blockade involves restored IL-2 production and proliferation of $\mathrm{CD}^{+} \mathrm{T}$ cells directly within the tumor microenvironment. J Immunother Cancer. 2, 114 (2014).

17. Wang HC, Chen CW, Yang CL, et al. Tumor-associated macrophages promote epigenetic silencing of gelsolin through DNA methyltransferase 1 in gastric cancer cells. Cancer Immunol Res. 5, 885-897 (2017).

18. Galon J, Costes A, Sanchez-Cabo F, et al. Type, density, and location of immune cells within human colorectal tumors predict clinical outcome. Science. 313, 1960-1964 (2006).

19. Nonomura N, Takayama H, Kawashima A, et al. Decreased infiltration of macrophage scavenger receptor-positive cells in initial negative biopsy specimens is correlated with positive repeat biopsies of the prostate. Cancer Sci. 101, 1570-1573 (2010).

20. Guo $\mathrm{C}, \mathrm{Yi} \mathrm{H}, \mathrm{Yu} X$, et al. In situ vaccination with CD204 gene-silenced dendritic cell, not unmodified dendritic cell, enhances radiation therapy of prostate cancer. Mol Cancer Ther. 11, 2331-2341 (2012).

21. Wiegel T, Bartkowiak D, Bottke D, et al. Adjuvant radiotherapy versus wait-and-see after radical prostatectomy: 10-year follow-up of the ARO 96-02/AUO AP 09/95 trial. Eur Urol. 66, 243-250 (2014). 
22. Hwang WL, Tendulkar RD, Niemierko A, et al. Comparison between adjuvant and early-salvage postprostatectomy radiotherapy for prostate cancer with adverse pathological features. JAMA Oncol. http:// doi: 10.1001/jamaoncol.2017.5230 (2018).

23. Hackman G, Taari K, Tammela TL, et al. Randomised Trial of Adjuvant Radiotherapy Following Radical Prostatectomy Versus Radical Prostatectomy Alone in Prostate Cancer Patients with Positive Margins or Extracapsular Extension. Eur Urol. 76, 586-595 (2019).

\section{Figures}
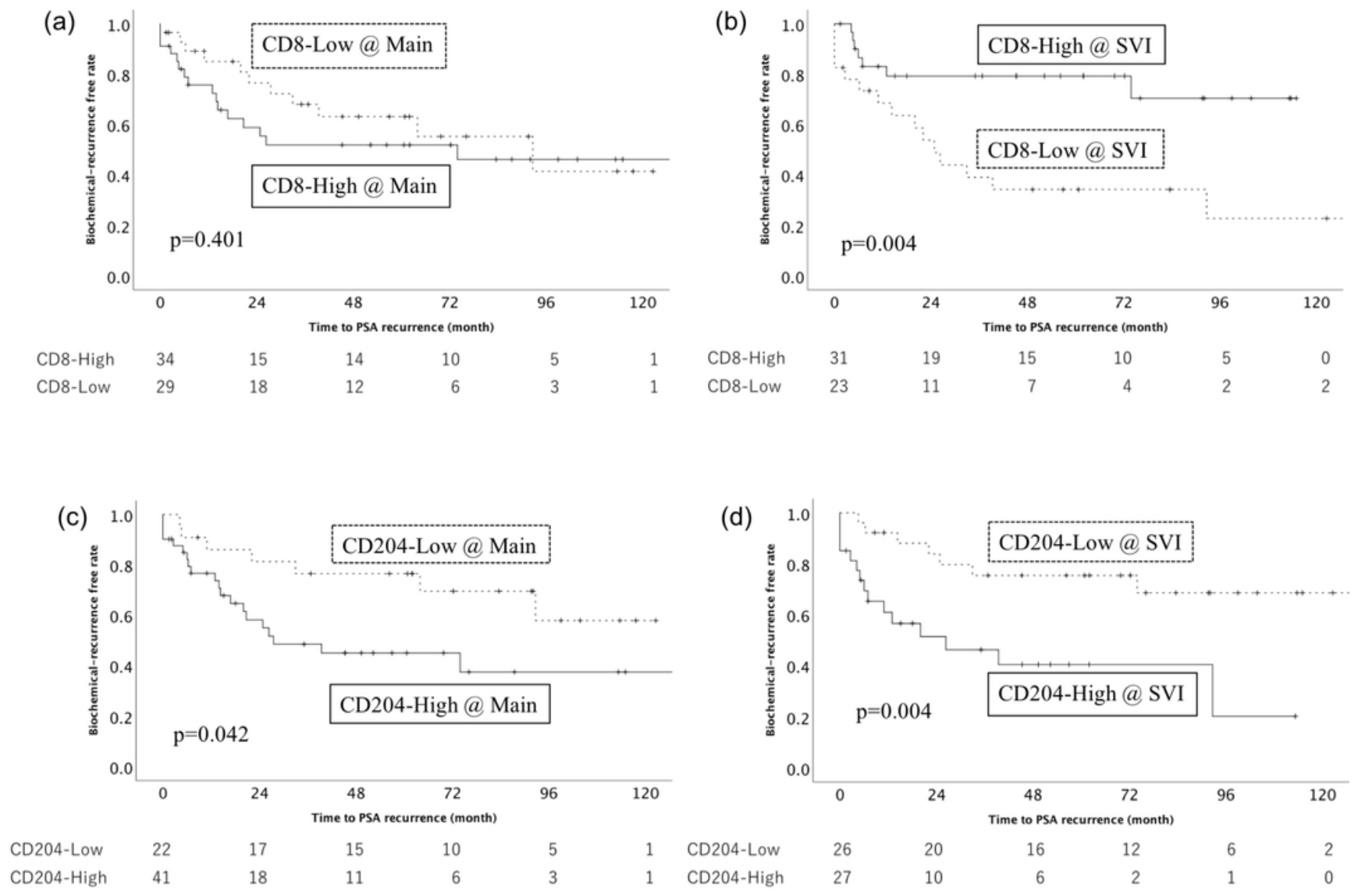

\section{Figure 1}

The Kaplan-Meier analysis of the recurrence-free survival of patients with seminal vesicle invasion according to the CD8+ cell in the main tumor area $(a, p=0.401)$ and seminal vesicle invasion area $(b$, $p=0.004)$ and the CD204+ cell in the main tumor area $(c, p=0.042)$ and seminal vesicle invasion area ( $d$, $p=0.004)$. Solid square means higher density, and dotted square means lower density. 

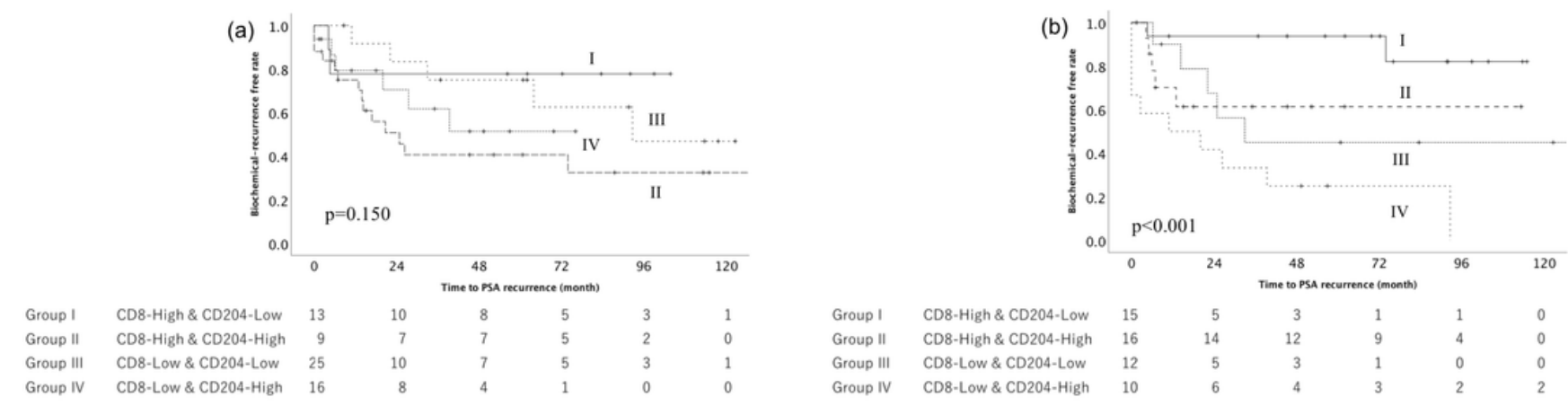

\section{Figure 2}

The Kaplan-Meier analysis of the recurrence-free survival of patients with seminal vesicle invasion according to the combination of the CD8+ and CD204+ cell. No significant difference between four groups was observed in the main tumor area $(a, p=0.150)$. However, a significant difference between four groups was observed in the seminal vesicle invasion area $(b, p<0.001)$. Group II, which included patients with the higher CD8+ cell count and the lower CD204+ cell count, had the most favorable outcome among the four groups. The 6-year progression free survival (PFS) rate of Group II was $90.9 \%$.
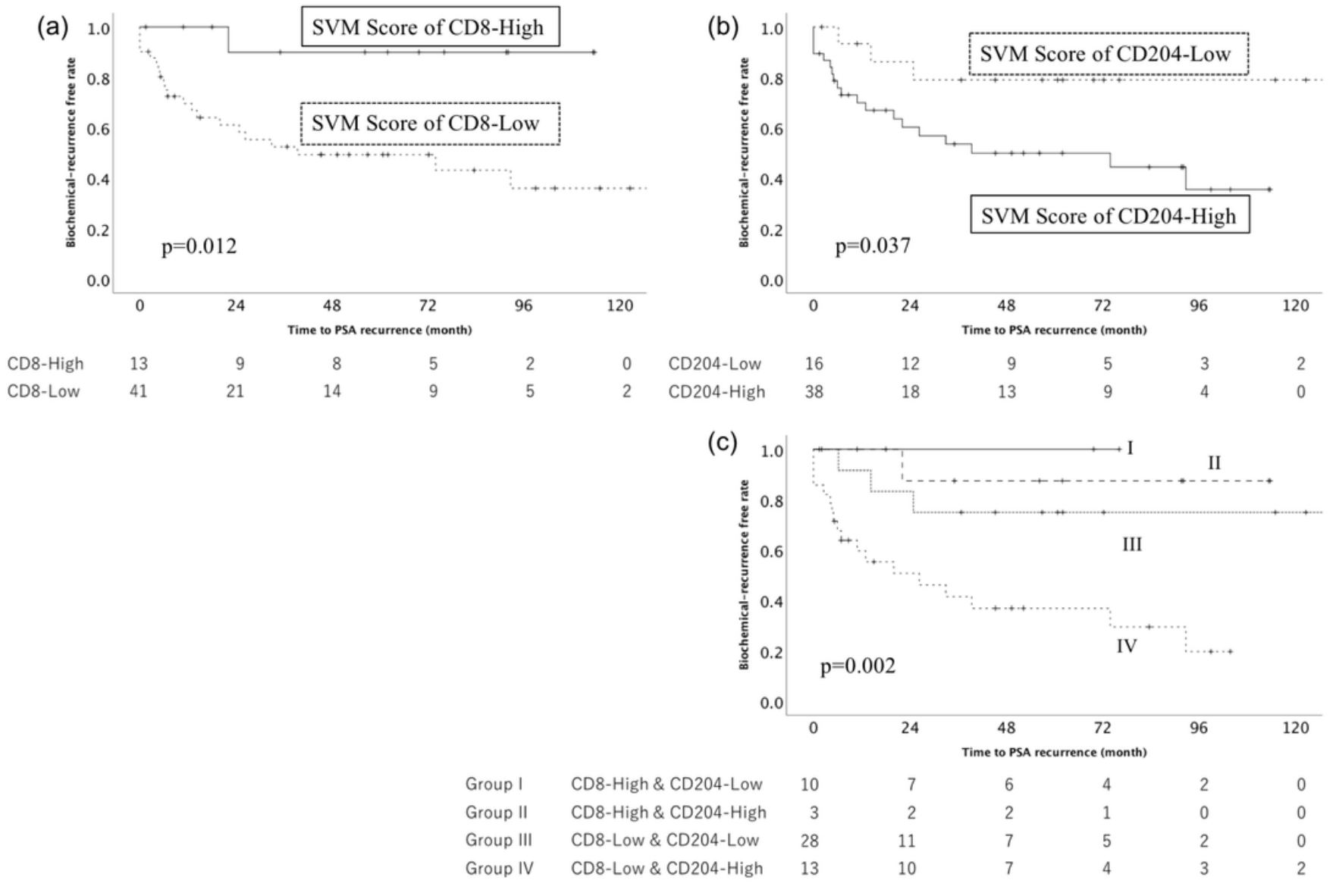


\section{Figure 3}

The Kaplan-Meier analysis of the recurrence-free survival of patients with seminal vesicle invasion according to the ratio of the CD8+ and CD204+ cells in the seminal vesicle invasion area to those in the main tumor area (SVM Score) for each tumor. A significant difference between the higher and lower SVM Score of CD8+ and CD204+ cell count was observed (CD8: $p=0.012,4 a, C D 204: p=0.037,4 b)$. Group I, which included patients with the higher SVM Score of CD8+ cell count and the lower SVM Score of CD204+ cell count, had the significantly favorable outcome $(p=0.002,4 c)$.

\section{Supplementary Files}

This is a list of supplementary files associated with this preprint. Click to download.

- SupFigure.pdf

- SupplementalFigurelegends.docx 